\title{
Graphene: A Peculiar Allotrope Of Carbon
}

\author{
Laxmi Nath Bhattarai \\ Department of Physics, Butwal Multiple Campus \\ Correspondence: lakshmibhattarai@gmail.com
}

\begin{abstract}
Graphene is a two dimensional one atom thick allotrope of Carbon. Electrons in grapheme behave as massless relativistic particles. It is a 2 dimensional nanomaterial with many peculiar properties. In grapheme both integral and fractional quantum Hall effects are observed. Many practical application are seen from use of Graphene material.
\end{abstract}

Keywords: Graphene, allotrope, Quantum Hall effect, nanomaterial.

\section{Introduction}

Graphene is a two dimensional allotropic form of carbon. This was discovered in 2004. Diamond and Graphite are three dimensional allotropes of carbon which are known from ancient time. One dimensional carbon nanotubes were discovered in 1991 and zero dimensional Fullerenes were discovered in 1985. Graphene was experimentally extracted from 3-dimensional Graphite by Physisists Andre Geim and Konstantin Novoselov of Manchester University UK in 2004. For their remarkable work they were awarded the Nobel Prize of Physics for the year 2010.

\section{Structure}

Graphene is a mono layer of Carbon atoms packed into a honeycomb crystal structure. Graphene sheets are one atom thick 2-dimensional layers of $\mathrm{sp}^{2}$ bonded carbon. In Graphene carbon atoms are arranged in Hexagonal structure with two atoms per unit cell. The Carbon - Carbon bond length in Graphene is $1.42 \mathrm{~nm}$ (Geim et al., 2007).

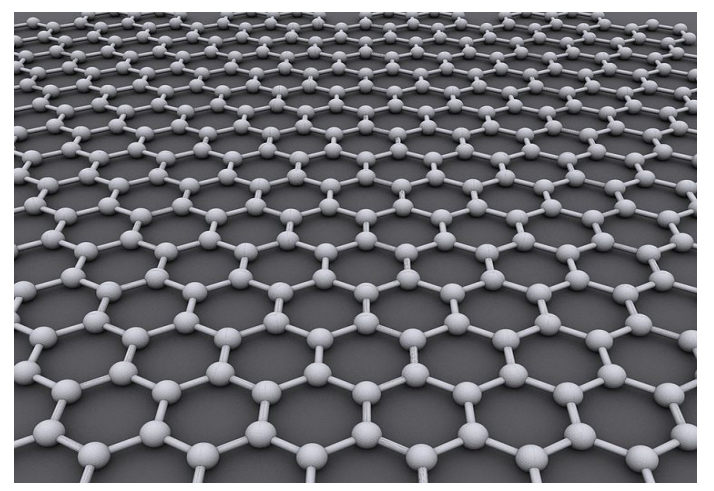

Fig: 1 Graphen un single molecular layer.

\section{Honey comb structure of Graphene}

Graphene is considered as the mother of all graphitic materials because it is the building block of carbon materials of all other dimensions (Srinivasan, 2007). Griphite is obtained by the stacking of Graphene layers, Diamond can be obtained from Graphene under extreme pressure and temperatures. One-dimensional carbon nanotubes can be obtained by rolling and Zero-dimensional Fullerenes is obtained by wrapping Graphene layers.

\section{Properties}

The young material Graphene is found to have following unique properties:

a) It is the thinnest material in the universe and the strongest ever measured in terms of Young's modulus and elastic stiffness. Graphene has breaking strength 200 times greater than steel with a tensile strength of $130 \mathrm{GPa}$ (Lee et al., 2008) and its Young's modulus is $0.5 \times 10^{12} \mathrm{Nm}^{-2}$ (Frank et al., 2007).

b) Graphene has massless charge particles. In Graphene the electrons behave as massless relativistic particles called as Dirac fermions. The speed of electrons is found to be $10^{6} \mathrm{~ms}^{-1}$. This velocity is called as Fermi velocity. Though their rest mass is not zero their relativistic mass vanishes in Graphene (Sahoo \& Das, 2009).

c) When the carrier concentration is zero Graphene exhibits a minimum conductivity of the order of $\mathrm{e}^{2} / \mathrm{h}$ where e is electronic charge and $\mathrm{h}$ is Planck constant whereas in other cases when the carrier 
charge concentration is zero the conductivity is also zero (Sahoo \& Dutta, 2010).

d) Near room temperature the thermal conductivity of Graphene lies between $(4.84 \pm 0.44) \times 10^{3}$ to $(5.30 \pm 0.48) \times 10^{3} \quad \mathrm{~W} \mathrm{~m}^{-1} \mathrm{~K}^{-1}$ which is 100 times larger than the conductivity of Graphite (Balandin et al., 2008). Graphene also beats diamond in thermal conductivity. Graphene now holds the record for conducting heat. It is better than any other known material.

e) Graphene has properties like both of metal and semiconductor. It can conduct electric current better than copper. It has the conduction and valence bands as in semiconductor but with no band gap. Hence it is also called as Semi-metal (Castro Neto, 2010) or a zero gap semiconductor or a hybrid between metal and semiconductor.

f) The resistivity of Graphene sheet is $10^{-6} \mathrm{ohm} \mathrm{cm}$. which is less than the resistivity of silver and is the lowest resistivity of known substances at room temperature (Sahoo \& Dutta, 2010).

g) Both integral and fractional quantum Hall effects are observed in Graphene at room temperature. Graphene shows the anomalous quantum hall effect with the sequence shifted by $1 / 2$ with reference to the standard sequence. This anomalous quantum Hall effect is the direct evidence for Dirac Fermions in Graphene (Sahoo, 2011).

\section{Applications}

Following applications are expected from Graphene :

a) In chemical and bio sensing devices.

b) Ultracapacitors made of Graphene composites would be capable of storing much larger amount of renewable energy from solar, wind and wave energy than that current technologies permit.

c) Graphene's high electrical conductivity and high optical transparency would make it a candidate for transparent conducting electrodes, required in applications in touch screens, liquid crystal displays, organic LED and organic photovoltaic cell.

d) Its ability to produce light is seen to improve Light Emitting Diodes (LEDs).

e) The amazing properties of graphene are seen as replacement for silicon semiconductors. It is expected to produce Transistors and Microchips of very small size with high operating speed. f) Graphene has a high carrier mobility, as well as low noise, allowing it to be used as the channel in a FET.

g) IBM researchers announced that they had succeeded in creating the first graphene- based integrated circuit, a broadband radio mixer. The circuit handled frequencies up to $10 \mathrm{GHz}$, and its performance was unaffected by temperatures up to $127^{\circ} \mathrm{C}$.

h) Its applications in future are seen as like plastic's today.

\section{Conclusion}

On the basis of various properties of graphene, it is regarded as a wonderful material whose application is seen in many fields of daily life. Therefore, this material is of great importance to develop new devices for mankind and is expected to open a new era in the field of scientific and technological development.

\section{References}

1. A Castro Neto, et al. (2009) Rev Mod Phys 81: 109.

2. Balandin, A. A., et al. (2008) Nano Letters ASAP 8 (3): 902-907.

3. Frank, I. W., Tanenbaum, D. M., Van Der Zande, A.M., \& McEuen, P. L.(2007) J. Vac. Sci. Technol. B 25 (6): 2558-2561.

4. Geim, A. K. \& Novoselov, K. S. (2007) Nature Materials $6(3): 183-191$

5. http://physics.bu.edu/document/pw1106.pdf.

6. http://wikipedia.org/wiki/Graphene, 1 July 2011 at 20:21.

7. Lee, C. et al. (2008) "Measurement of the Elastic Properties and Intrinsic Strength of Monolayer Graphene". Science 321 (5887): 385.

8. Sahoo S. \& Das S.(2009) Indian Journal of Pure and Applied Physics, 47,186.

9. Sahoo S. \& Dutta, A.K.(2010) Physics Education, July -September, 207-213.

10. Srinivasan, C., (2007) Current Science, 92, 1338. 